\title{
Parasitic Myoma Identified 14 Years After Laparoscopic Myomectomy
}

\author{
Akihiro Takeda, MD, Shotaro Hayashi, MD, Sanae Imoto, MD, Hiromi Nakamura, MD \\ Department of Obstetrics and Gynecology, Gifu Prefectural Tajimi Hospital, Tajimi, Gifu, Japan (all authors).
}

\begin{abstract}
Introduction: Parasitic myoma after laparoscopic myomectomy with power morcellation is an emerging concern that has an iatrogenic aspect. However, the duration necessary for observation to identify parasitic myoma has not yet been clarified.

Case Description: A 47-year-old, gravida 2, para 2 woman was referred for an unusual pelvic mass. Her gynecological history was significant, with laparoscopic myomectomy with power morcellation for intraligamental myoma 14 years earlier. Uterine myoma, endometrial polyp, and suspicious parasitic myomas were diagnosed by magnetic resonance imaging. In a laparoscopic view, 4 parasitic myomas were identified. Single-port laparoscopic-assisted vaginal hysterectomy, bilateral salpingo-oophorectomy, and debulking of the parasitic myomas were performed. Histopathological examination demonstrated that the parasitic myomas were histologically similar to the myomas excised 14 years earlier.
\end{abstract}

Conclusion: Women who have already undergone myoma surgery with power morcellation should be followed up carefully for the potential development of parasitic myoma.

Key Words: Laparoscopic myomectomy, Magnetic resonance imaging, Parasitic myoma, Power morcellation.

Citation Takeda A, Hayashi S, Imoto S, Nakamura H. Parasitic myoma identified 14 years after laparoscopic myomectomy. CRSLS e2015.00076. DOI: 10.4293/CRSLS.2015.00076.

Copyright (c) 2015 by SLS, Society of Laparoendoscopic Surgeons. This is an open-access article distributed under the terms of the Creative Commons Attribution-Noncommercial-ShareAlike 3.0 Unported license, which permits unrestricted noncommercial use, distribution, and reproduction in any medium, provided the original author and source are credited.

Disclosure: The authors report no conflicts of interest. The authors have no relationships with the companies that may have a financial interest in the information contained in the manuscript.

Address correspondence to: Akihiro Takeda, MD, Department of Obstetrics and Gynecology, Gifu Prefectural Tajimi Hospital, 5-161 Maebata-cho, Tajimi, Gifu 507-8522, Japan. Telephone: +81-572-22-5311, Fax: +81-572-25-1246, E-mail: gyendoscopy@gmail.com

\section{INTRODUCTION}

Advances in surgical equipment and techniques have facilitated laparoscopic myomectomy for symptomatic myoma in women desiring minimally invasive management. ${ }^{1}$ However, this procedure requires the removal of excised myoma tissue through a small incision by fragmentation in the abdominal cavity. ${ }^{1}$

Although the introduction of an electromechanical power morcellator significantly shortens the surgical process for specimen retrieval, ${ }^{2}$ a power morcellator can produce viable myoma particles that may eventually disseminate within the peritoneal cavity. ${ }^{3}$ In limited instances when minute myoma particles survive and become implanted on the tissue surface of the peritoneal cavity, iatrogenic parasitic myoma may eventually develop. ${ }^{4-11}$ Thus, for women who have already undergone myoma surgery with power morcellation, it is important to determine the appropriate duration of follow-up necessary to identify the potential development of parasitic myoma. ${ }^{5}$

Herein, we describe a case of parasitic myoma diagnosed 14 years after the patient had undergone laparoscopic myomectomy with power morcellation. Institutional review board approval of Gifu Prefectural Tajimi Hospital and informed consent of the patient were obtained to report this case.

\section{CASE REPORT}

A 47-year-old, gravida 2, para 2 woman was referred for an unusual pelvic mass found at regular checkup by transvaginal ultrasonography. Her gynecological history was significant, for laparoscopic myomectomy with 

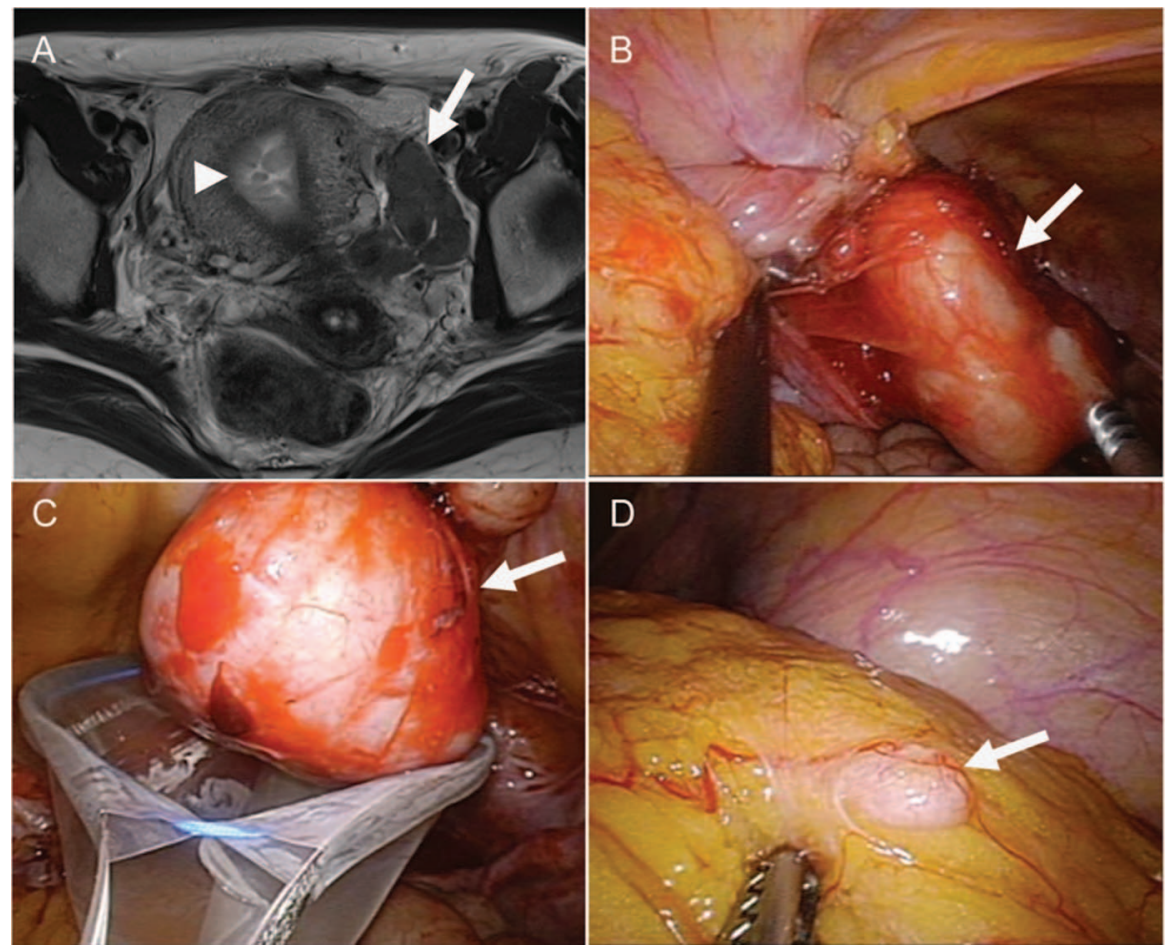

Figure 1. (A) T2-weighted MRI showing the parasitic myoma (arrow) with multiple endometrial polyps (arrowhead) in the uterine cavity. (B) Single-port laparoscopic view showing a parasitic myoma growing on the surface of the left pelvic peritoneum (arrow). (C) Excised parasitic myoma (arrow) put into a retriever bag for extraction. (D) Small parasitic myoma (arrow) growing on the surface of the major omentum.

power morcellation for intraligamental myoma 14 years earlier. On axial T2-weighted magnetic resonance imaging (MRI) (Figure 1A), a mass suspicious for parasitic myoma in the left adnexal region (arrow), an intrauterine lesion with possible multiple endometrial polyps (arrowhead), and an intramural myoma were identified.

After informed consent was obtained from the patient and her husband, single-port laparoscopic-assisted vaginal hysterectomy and bilateral salpingo-oophorectomy were performed. The parasitic myoma attached to the left pelvic peritoneum (Figure 1B, arrow) was then excised by blunt dissection, with coagulation and cutting of the feeding vessel with Ligasure V (Covidien Japan, Tokyo, Japan). The excised tissue (Figure 1C, arrow) was put into a retriever bag and carried out of the body through the umbilical incision after fragmentation with a cold scalpel inside the bag. Then, a small parasitic myoma growing on the omental surface was identified (Figure 1D, arrow). After the omentum had been pulled out of the body through the umbilical port, a partial omentectomy was extracorporeally performed. Finally, 2 peritoneal parasitic myomas with a diameter of 5 and $3 \mathrm{~cm}$ each and 2 omental parasitic myomas with diameter of $0.5 \mathrm{~cm}$ each were retrieved.

Histopathological examination demonstrated that these parasitic myomas (Figure 2B) were similar to the myoma tissue excised 14 years earlier (Figure 2A). The postoperative course was uneventful. Evidence of tumor recurrence has not been noted a year after surgery.

\section{DISCUSSION}

The laparoscopic power morcellator was once thought of as a significant advance in avoiding major laparotomy for specimen retrieval during laparoscopic myomectomy for many women with symptomatic uterine myoma. ${ }^{2}$ However, its use has now raised concerns, including parasitic myoma, which can adversely affect a woman's health for a long time. ${ }^{3-11}$

Parasitic myoma is a rare form of late complication after power morcellation, with a reported prevalence of $0.4 \% \%^{8}$ and $1.2 \%$ in large case series. However, because it may occur years after power morcellation, it seems reasonable 

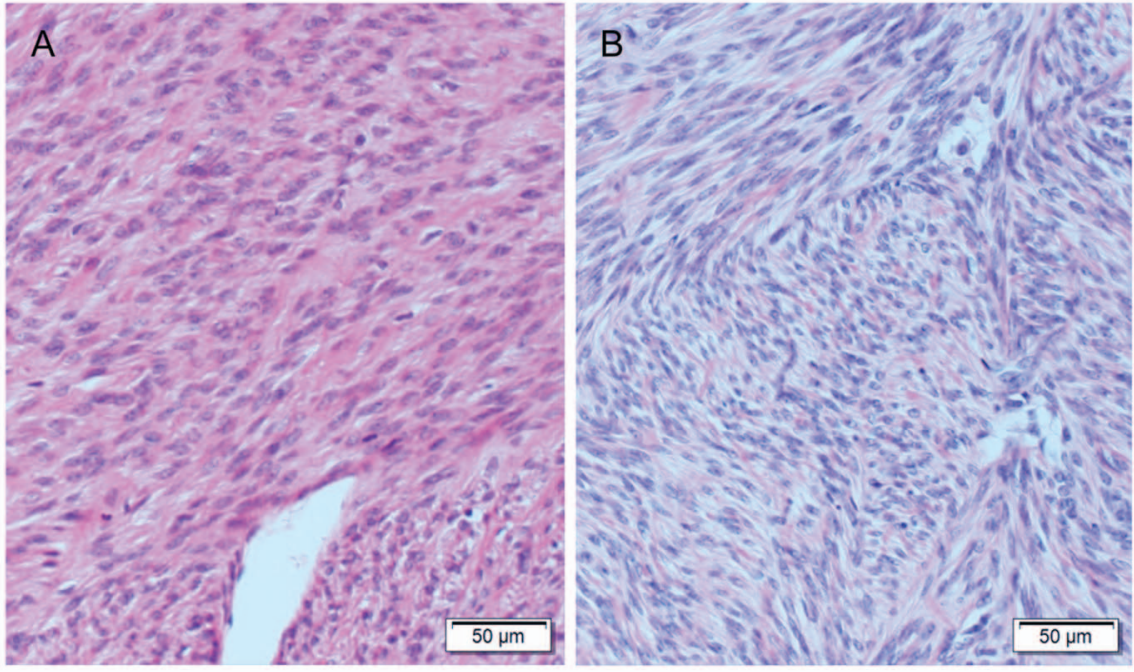

Figure 2. Histological findings in the myoma tissue. (A) Myoma tissue resected in the initial laparoscopic myomectomy with power morcellation. (B) Recurrent parasitic myoma tissue resected 14 years after the initial myomectomy with power morcellation. Hematoxylin-eosin.

to speculate that a significant number of cases have remained undiagnosed because the patient was lost to follow-up, was asymptomatic, or was receiving treatment elsewhere.

In addition to determining the true incidence of parasitic myoma, an important concern is clarifying the duration of follow-up necessary to identify the occurrence of parasitic myoma. If parasitic myoma possesses progesterone dependence similar to that of ordinary uterine myoma, ${ }^{12}$ follow-up could be terminated by the time of surgical or spontaneous menopause. However, if there is a possibility that presently unknown altered cellular characteristics are acquired during the process of parasitic myoma formation, follow-up for a longer period may be necessary, even after menopause.

Upon reviewing 11 cases reported in the literature, ${ }^{4-11}$ with precise descriptions of the use of a power morcellator for myoma extraction, the median duration of parasitic myoma development after power morcellation was 5 years (range, 0.9-9 years). However, the present case indicates that a longer observational period is needed to identify the health problems related to parasitic myoma.

The natural history and developmental mechanism of parasitic myoma after power morcellation are not well understood. ${ }^{4-11}$ Even though the loss of viable tissue fragments within the abdominal cavity during power morcellation could be responsible for the later devel- opment of parasitic myomas, such particles, which are devoid of blood supply, would be mostly reabsorbed in the peritoneal cavity. On the other hand, if retained fragments establish a blood supply from the peritoneal surface, implantation and subsequent growth of parasitic myoma can occur anywhere in the peritoneal cavity.

During the implantation process, in addition to the simple attachment and growth of myoma particles, other stimulatory factors such as progesterone may facilitate attachment and growth by promoting neovascularization, ${ }^{5}$ even from small myoma fragments, as shown in the present case report.

The diagnosis of retained myoma fragments forming parasitic myoma is challenging both clinically and radiologically, because most patients may be asymptomatic or have nonspecific initial symptoms such as pelvic pain and dyspareunia. ${ }^{4-11}$ Therefore, in women with a history of laparoscopic morcellation who report new or recurrent pelvic symptoms or present with a new pelvic mass, iatrogenic parasitic myomas should be included for the differential diagnosis as a late complication of power morcellation. However, the finding of an unusual pelvic mass by ultrasonography warrants the exclusion of a malignant condition, and further diagnostic examination by MRI, because of its superior space-resolving power, would be necessary before determining the appropriate management procedure.

In conclusion, long-term follow-up is necessary to identify parasitic myoma after laparoscopic myomectomy with 
power morcellation, to protect women's health and to clarify further the precise incidence and developmental process of this condition.

\section{References:}

1. Hurst BS, Matthews ML, Marshburn PB. Laparoscopic myomectomy for symptomatic uterine myomas. Fertil Steril. 2005;83: $1-23$.

2. Steiner RA, Wight E, Tadir Y, Haller U. Electrical cutting device for laparoscopic removal of tissue from the abdominal cavity. Obstet Gynecol. 1993;81:471-474.

3. Senapati S, Tu FF, Magrina JF. Power morcellators: a review of current practice and assessment of risk. Am J Obstet Gynecol. 2015;212:18-23.

4. Paul PG, Koshy AK. Multiple peritoneal parasitic myomas after laparoscopic myomectomy and morcellation. Fertil Steril. 2006;85:492-493.

5. Takeda A, Mori M, Sakai K, Mitsui T, Nakamura H. Parasitic peritoneal leiomyomatosis diagnosed 6 years after laparoscopic myomectomy with electric tissue morcellation: report of a case and review of the literature. J Minim Invasive Gynecol. 2007;14: $770-775$.

6. Kumar S, Sharma JB, Verma D, Gupta P, Roy KK, Malhotra N. Disseminated peritoneal leiomyomatosis: an unusual complication of laparoscopic myomectomy. Arch Gynecol Obstet. 2008; 278:93-95.
7. Epstein JH, Nejat EJ, Tony Tsai T. Parasitic myomas after laparoscopic myomectomy: case report. Fertil Steril. 2009;91: 932.e13-e14.

8. Paul PG, Naik SA, Madhu KN, Thomas T. Complications of laparoscopic myomectomy: a single surgeon's series of 1001 cases. Aust N Z J Obstet Gynaecol. 2010;50:385-390.

9. Cucinella G, Granese R, Calagna G, Somigliana E, Perino A. Parasitic myomas after laparoscopic surgery: an emerging complication in the use of morcellator?-description of four cases. Fertil Steril. 2011;96:e90-e96.

10. Huang PS, Chang WC, Huang SC. Iatrogenic parasitic myoma: a case report and review of the literature. Taiwan J Obstet Gynecol. 2014;53:392-396.

11. Yoshida A, Nii S, Matsushita H, Morii Y, Watanabe K, Wakatsuki A. Parasitic myoma in women after laparoscopic myomectomy: a late sequela of morcellation? J Obstet Gynaecol. 2015;35: 322-323.

12. Ishikawa H, Ishi K, Serna VA, Kakazu R, Bulun SE, Kurita T. Progesterone is essential for maintenance and growth of uterine leiomyoma. Endocrinology. 2010;151:2433-2442. 